\title{
Output Speed Prediction of an Overrunning Clutch for Oscillatory Input Motion using a Simplified Approach
}

\author{
M. M. Mokhlis ${ }^{1, a}$ and M. B. Baharom ${ }^{1}$ \\ ${ }^{1}$ Department of Mechanical Engineering, Universiti Teknologi PETRONAS, Malaysia
}

\begin{abstract}
This paper presents the derivation of a simplified mathematical equation to predict the output speed of an overrunning clutch. The working principle of the overrunning clutch was studied and mathematical equations were derived for different cases. A MATLAB Simulink model was created to simulate the equations and ADAMS/View simulation was used for verification. The equations are able to calculate the output motion with minimal errors. The equation can be used for a quick estimation to predict the output speed for any kind of input motions.
\end{abstract}

\section{Introduction}

Overrunning clutch is an essential component that can be found in various mechanical systems such as bicycle, engine starter or engine transmission. The component functions to rectify the transmission of torque and speed into one direction and prevent the transmission in reverse direction. T. Xu and G.G Lowen had derived a differential equation for a sprag-type overrunning clutch to determine the output motion based on the input motion with a known external load [1]. The equation contains the sprag and race inertia, Hertzian contact force and non-linear damping model. The equation could provide an accurate output motion value but would be tedious to be utilized for a quick prediction of output motion.

Overrunning clutch in freewheel (disengage) operation can be regarded to have similar behavior to a bearing. For a typical bearing, the friction torque can be calculated from the summation of rolling friction, sliding friction, seal friction and drag loss [2]. However, the friction torque is also affected by the motion speed where higher speed will create higher friction torque. For a standard operation in a dry environment, the major components of the friction torque are the rolling and sliding friction. $\mathrm{K}$. Liu et al. had done a study on the effect of the sliding friction on the overrunning clutch with an oscillatory input motion [3]. The study shows that a well lubricated clutch will reduce the sliding friction. Furthermore, smaller roller radius and higher number of roller will also reduce the friction. In addition, they also had done a study on the effect of the rolling friction based on the number and size of the rollers [4]. The study claimed that the rolling friction torque is directly proportional to the input load with a power of $3 / 2$. Both of the studies showed that the friction torque is lower for medium to high speed compared to low speed for the case of oscillating input motion. Similarly, K. Liu et al. had done another study on the rolling friction which stated that the friction occurred due to elastic, plastic hysteresis, viscous shear and micro slipping [5].

\footnotetext{
a mohaimin.mokhlis@gmail.com
}

This is an Open Access article distributed under the terms of the Creative Commons Attribution License 2.0, which permits unrestricted use, distribution, and reproduction in any medium, provided the original work is properly cited. 
The slipping of the input race when engaging with the output race will cause a lag to the power transmission. The scenario is caused by the slipping torque during the engagement process. YC Chen et al. showed that the slipping torque can be influenced by the design parameter of the overrunning clutch [6]. The torque will increase if the friction coefficient increase, the roller radius tolerance decrease or the number of roller increases. Because of that, there will be a dynamic locking where the output race will accelerate to the input race speed at the moment of engagement. K. Liu et al. had focus on the matter and a profile of dynamic load versus engaging time was made [7]. In addition, the slip angle was able to be calculated from the result. CD Hua et al. noticed that the load has impact on the overrunning clutch performance [8]. The friction lock up is related to the geometrical shape of the contacting components, material characteristic, loading capacity and contacting state.

In this paper, a simplified method to predict the output speed of the overrunning clutch is formulated. The study is focused on an oscillatory type of input speed where forward and backward motions are transmitted to the overrunning clutch to produce a single direction output speed. Due to the repeating engagement and disengagement of the clutch, the output speed is expected to form a repeating pattern where the speed will drop during disengagement and speed rise during engagement. The equation is to be derived with the assumption that the slipping torque and dynamic locking can be neglected and the friction torque is constant throughout the motion speed. The validity range of the simplified method approach will be investigated as a function of moment of inertias.

\section{Mathematical Equations for Output Speed}

$\mathrm{K}$. Liu et al. have described the working principle of overrunning clutch for the case of a reciprocating motion input $[9,10]$. They stated that the clutch will convert the reciprocating input motion into a onedirectional rotational output. The planet wheel and the outer rim will disengage if the driver (input race or cage) rotates in the opposite direction or if the driven member (output race or hub) has a faster angular velocity.

Based on the working principle of the overrunning clutch, the operation can be derived into a few forms of mathematical equations. The equation consists of 3 cases depending on the differences between input and output speed. The input speed is denoted by $\omega_{\mathrm{i}}$ and output speed is denoted by $\omega_{\mathrm{o}}$.

\subsection{Case 1: Engage (Lock)}

$$
\omega_{\mathrm{o}}=\omega_{\mathrm{i}} \text { when } \omega_{\mathrm{o}}<\omega_{\mathrm{i}}
$$

Engagement only occurs when the input speed is higher than the output speed.

\subsection{Case 2: Disengagement (Freewheel) During Idle}

$$
\omega_{\mathrm{o}}=0 \text { when } \omega_{\mathrm{i}}<0 \& \omega_{\mathrm{o}}=0
$$

When the hub is in the stationary position (idle), if the input speed rotates in reverse direction, the output cage speed will remain stationary.

\subsection{Case 3: Disengagement (Freewheel) During Operation}

$$
\omega_{\mathrm{o}}=\omega_{\mathrm{o}(\mathrm{t}-1)}+\alpha t \text { when } \omega_{\mathrm{o}}>\omega_{\mathrm{i}} \& \omega_{\mathrm{o}}>0
$$

During disengagement, the output speed will drop due to the angular acceleration which can be calculated from the following equation:

$$
T_{\mathrm{ex}}-T_{\mathrm{f}}=\mathrm{I} \alpha
$$

Where $\mathrm{T}_{\mathrm{ex}}$ is applied torque, $\mathrm{T}_{\mathrm{f}}$ is friction torque, $\mathrm{I}$ is moment of inertia and $\alpha$ is angular acceleration. During disengagement, the applied torque is zero which leads to the formulation of angular acceleration as follows:

$$
\alpha=-T_{\mathrm{f}} / \mathrm{I}
$$


Based on Eq. (5), it is clear that if $\mathrm{T}_{\mathrm{f}}$ is small, $\alpha$ will be small as well and this will give a small output speed drop. Minimal speed drop will ensure that the speed variation is low. The inertia is inversely proportional to the speed drop. Therefore it is desirable to have higher inertia to maintain the output speed. The graphical representation of the previous three cases can be represented in Figure 1.

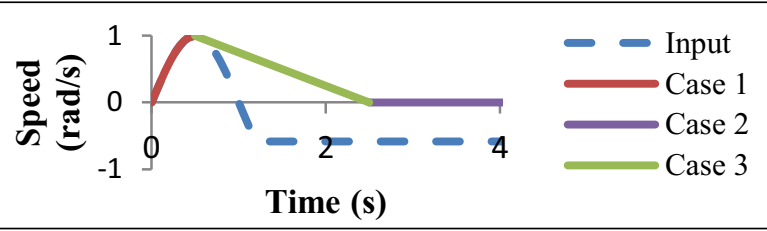

Figure 1. Graphical Representation of different cases of overrunning clutch operations

\section{Modeling and Simulation}

\subsection{MATLAB Model}

A MATLAB Simulink model was created based on the mathematical equations. The model is suitable to predict the output speed for a specific friction torque and moment of inertia. Figure 2 shows the MATLAB Simulink model.

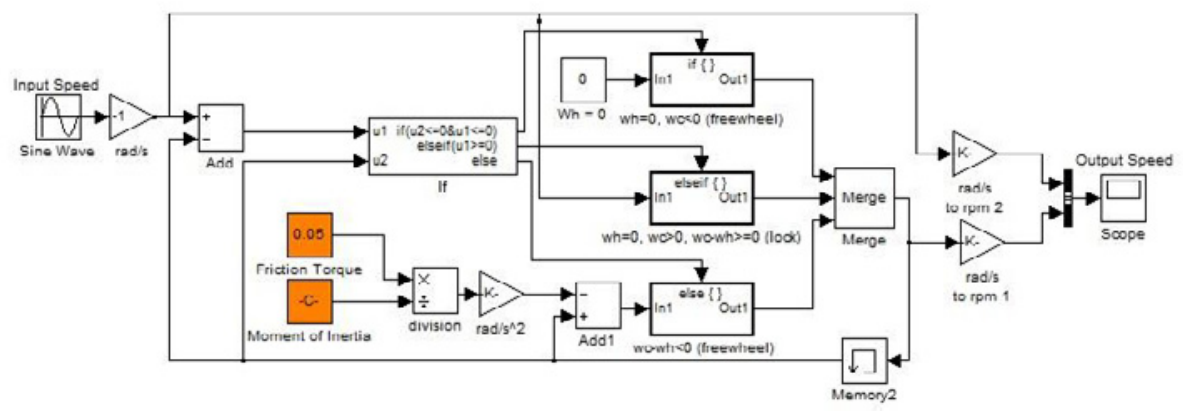

Figure 2. MATLAB Simulink Model

The model was used to simulate different values of moment of inertias at a constant friction torque having the value of $0.05 \mathrm{Nm}$. The value was chosen from a typical overrunning clutch having an inner land outer diameter of about $30 \mathrm{~mm}$ and $60 \mathrm{~mm}$ respectively [11]. The input speed used is in the form of sinusoidal motion with the amplitude of $104.72 \mathrm{rad} / \mathrm{s}(1000 \mathrm{rpm})$ and frequency of $100 \mathrm{rad} / \mathrm{s}$. Figure 3 shows the resultant output speed for different inertias. The result shows that the speed variation is minimal when the value of inertia is high.

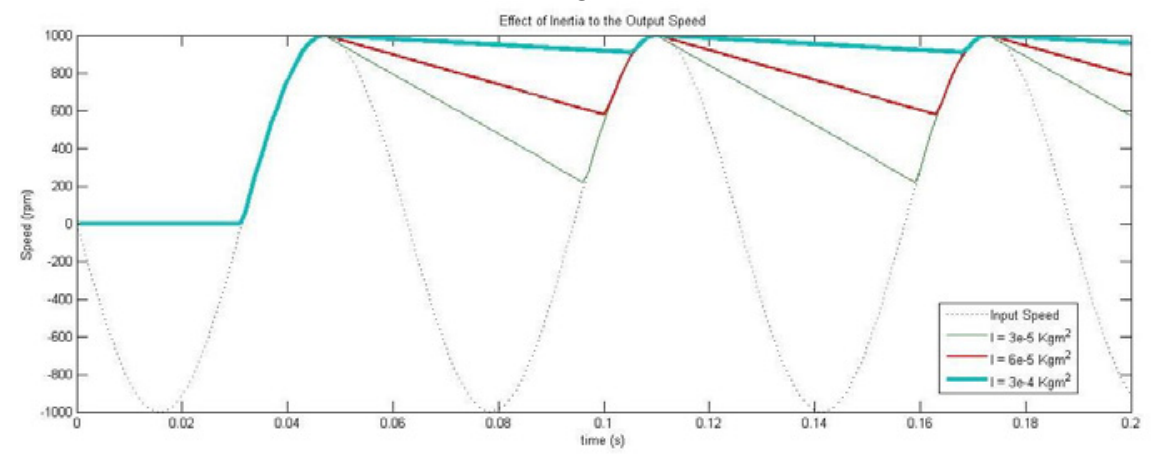

Figure 3. Effect of the Inertia to the Output Speed 


\subsection{ADAMS/View Model}

An ADAMS model was created to represent the real mechanism in order to determine the validity of the simplified mathematical models. Figure 4 shows the outline of an overrunning clutch which was modeled in ADAMS. The parameters used for the ADAMS model is shown in Table 1. The same input speed and friction torque from MATLAB Simulink are used and the values of the inertias are varied. The total inertia (I) is the summation of shaft inertia $\left(\mathrm{I}_{\mathrm{s}}\right)$, disc inertia $\left(\mathrm{I}_{\mathrm{d}}\right)$ and the hub inertia $\left(\mathrm{I}_{\mathrm{o}}\right)$. The disc inertia represents the load that the overrunning clutch is carrying. The shaft and hub inertia have a constant value throughout the simulation and only the disc inertia is varied.

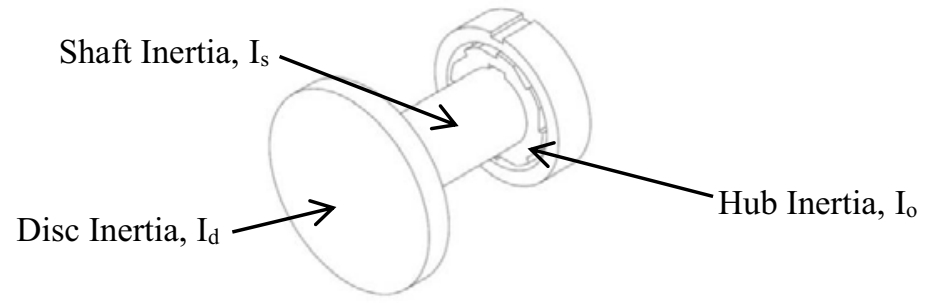

Figure 4. Model of an Overrunning Clutch with attached Disc

Table 1. Design Parameter of Overrunning Clutch model in ADAMS

\begin{tabular}{|c|c|}
\hline Parameter & Value \\
\hline Clutch Inner Diameter & $30 \mathrm{~mm}$ \\
\hline Clutch Outer Diameter & $62 \mathrm{~mm}$ \\
\hline Hub Outer Diameter & $46 \mathrm{~mm}$ \\
\hline Shaft Length & $80 \mathrm{~mm}$ \\
\hline
\end{tabular}

\section{Results and Discussion}

Figure 5 shows the result comparison between ADAMS and MATLAB for one value of moment of inertia. The graph shows that both results have the same pattern but with slight error.

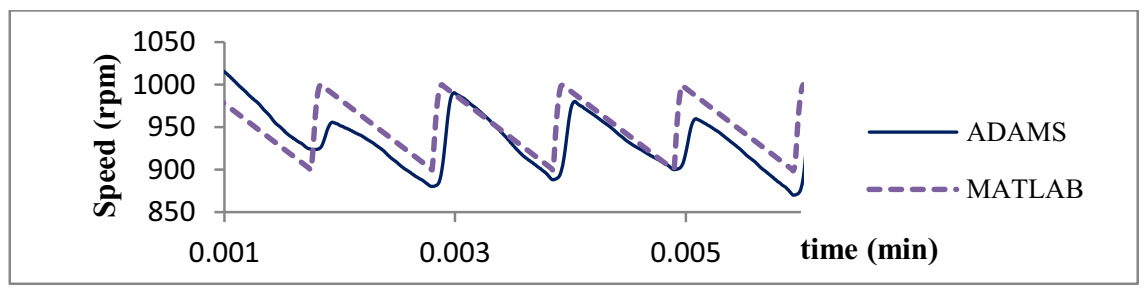

Figure 5. Result Comparison between ADAMS and MATLAB

Figure 6 shows the graph of the percentage of error in average value of speed between the ADAMS and MATLAB Simulink data versus the dimensionless ratio of total inertia, I to the hub inertia, $\mathrm{I}_{0}$. The ADAMS model was initially validated for the case of $\mathrm{I}=\mathrm{I}_{0}$ with error of about $5 \%$.

For the range of $\mathrm{I} / \mathrm{I}_{0}$ between 1 to 1.5 , the error is about 1 to $5 \%$. However, the results are not very significant because this simply means that the overrunning clutch does not carry any load.

For the range 1.5 to 10 , the error is about 3 to $14 \%$. The high percentage of error shows that even though the inertia is increasing, the drop in speed is still high. In addition, as ADAMS result is the actual result of the overrunning clutch's operation, the result consists of the slipping torque and dynamic locking in the simulation. This causes the percentage error to be high as it affects the performance of the clutch. 
For the ratio higher than 10 , the error is approximately between $2-7 \%$. With higher inertia, the speed drop variation will become smaller to a point that a constant speed can be achieved. When the inertia increases, the error value becomes steadier. This shows that the equations are good for predicting of the speed for high inertia with error up to $7 \%$.

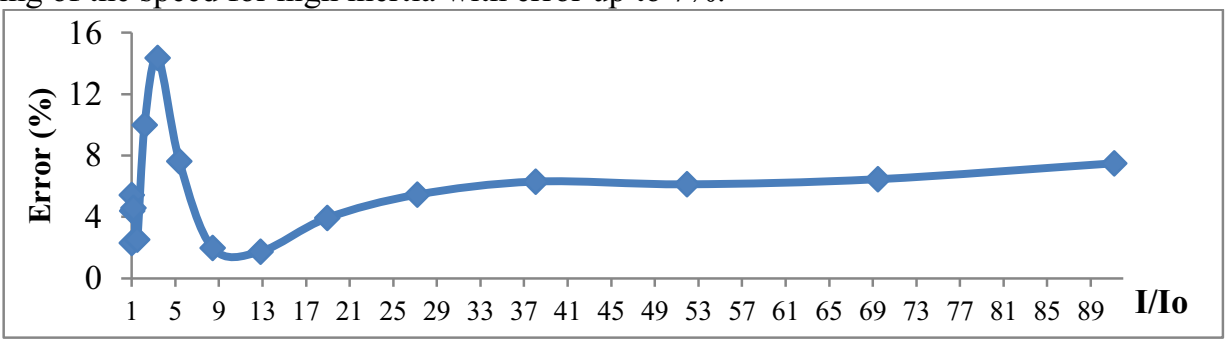

Figure 6. Graph of Error Percentage between ADAMS and MATLAB data versus I/Io

\section{Conclusion}

A simplified set of mathematical equations to predict the output motion of an overrunning clutch has been successfully derived and verified. The equations are able to give a good prediction of the output speed with low percentage of error $(2-7 \%)$ for inertia ratio higher than 10 . The derived equations are very useful in predicting the output speed of an overrunning clutch from an oscillating input speed during preliminary design stage of mechanisms. It can provide quick estimated results without involving rigorous calculations. The equation should also be able to be used for any kinds of overrunning clutches.

\section{References}

1. T. Xu, G.G Lowen. (2010). A Mathematical Model of an Over-Running Sprag Clutch. Mechanism and Machine Theory, 29(1), 11-23

2. SKF. (n.d). The SKF Model for Calculating the Frictional Moment. Retrieved October 11, 2013 from http:/www.skf.com/group/products/bearings-units-housings/ballbearings/principles/friction/skf-model/index.html

3. K. Liu, E. Bamba. (2005). Analytical Model of Sliding Friction in an Overrunning Clutch. Tribology International, 38(2), 187-194.

4. K. Liu, E. Bamba. (2005). Frictional Dynamics of the Overrunning Clutch for Pulse-Continuously Variable Speed Transmission: Rolling Friction. Wear, 217(2), 208-214.

5. K. Liu, Z. Haiyan. (1997). Research on Rolling Friction of the Overrunning Clutch. Journal of $X i$ 'an University of Technology, Issue 3. Retrieved from http://en.cnki.com.cn/Article_en/CJFDTOTAL-XALD199702002.htm

6. Y. C. Chen, L. W. Chen. (2006). Effect of Design Parameters on the Slipping Torque of an Overrunning Clutch. Journal of Automobile Engineering, 220(5), 563-570.

7. Liu, K., Zhang, H., Bamba, E. (1998). Dynamic Analysis of an Overrunning Clutch for the PulseContinuously-Variable-Speed Transmission. SAE Technical Paper, (980827).

8. C. D. Hua, J. W. Ping, M. Tanaka, S. Guizhi. (2007). Study of Contacting Friction and Load Bearing Performance of One-Way Clutch. Lubrication Engineering, Issue 3. Retrieved from http://en.cnki.com.cn/Article_en/CJFDTOTAL-RHMF200703057.htm

9. K. Liu, E. Bamba. (2005). Analytical Model of Sliding Friction in an Overrunning Clutch. Tribology International, 38(2), 187-194.

10. K. Liu, E. Bamba. (2005). Frictional Dynamics of the Overrunning Clutch for Pulse-Continuously Variable Speed Transmission: Rolling Friction. Wear, 217(2), 208-214.

11. RENOLD. (2010). Freewheels, Sprag and Trapped Roller Clutches. Retrieved December 30, 2013, from http://www.renold.com/nmsruntime/saveasdialog.asp?1ID=2060\&sID=4062 